\title{
CONTINUOUS SELECTION THEOREM, COINCIDENCE THEOREM AND INTERSECTION THEOREMS CONCERNING SETS WITH $H$-CONVEX SECTIONS
}

\author{
XIE-PING DING \\ (Received 31 January 1990; revised 30 June 1990)
}

Communicated by J. H. Rubinstein

\begin{abstract}
A continuous selection and a coincidence theorem are proved in $H$-spaces which generalize the corresponding results of Ben-El-Mechaiekh-Deguire-Granas, Browder, Ko-Tan, Lassonde, Park, Simon and Takahashi to noncompact and/or nonconvex settings. By applying the two theorems, some intersection theorems concerning sets with $H$-convex sections are obtained which generalize the corresponding results of Fan, Lassonde and Shih-Tan to $H$-spaces. Some applications to minimax principle are given.

1991 Mathematics subject classification (Amer. Math. Soc.) 54 C 65, 54 H 25, 52 A 07.

Keywords and phrases: Continuous selection, coincidence, $H$-space, contractible, weakly $H$ convex, $H$-compact, $H-K K M$, compactly closed (open), upper semi-continuous, minimax principle.
\end{abstract}

\section{Introduction}

In our recent papers [7, 9], we have obtained some new matching theorems, fixed point theorems and minimax inequalities. By applying a minimax inequality in [7], some non-convex generalizations of well-known intersection theorems concerning sets with convex sections were proved in [8], but we would have to assume that the product space is a $H$-space.

In the present paper, we shall first show a continuous selection theorem, an $H-K K M$ theorem and a coincidence theorem which improve and generalize

This project was supported by the National Natural Science Foundation of China.

(c) 1992 Australian Mathematical Society 0263-6115/92 \$A2.00+0.00 
the corresponding results of Ben-El-Mechaiekh-Deguire-Granas [4], Browder [6], Ding-Tan [10], Ko-Tan [16], Lassonde [17], Park [19], Simon [20], and Takahashi [23] to noncompact and nonconvex settings. Next by applying our earlier results, some intersection theorems concerning sets with $H$-convex sections are obtained without the assumption that the product space is a $\mathrm{H}$ space. These theorems generalize those of Fan [10, 12], Lassonde [17] and Shih-Tan [22] to noncompact and nonconvex settings. Some applications are given.

\section{Preliminaries}

Let $X$ be a nonempty set; we shall denote by $2^{X}$ the family of all subsets of $X$ and by $\mathscr{F}(X)$ the family of all nonempty finite subsets of $X$. Also $\Delta_{n}$ is the standard $n$ dimensional simplex with the vertices $e_{0}, e_{1}, \ldots, e_{n}$. If $J$ is a nonempty subset of $\{0, \ldots, n\}, \Delta_{J}$ will denote the convex hull of the vertices $\left\{e_{j}: j \in J\right\}$. Let $X$ and $Y$ be topological spaces and $D$ be a subset of $X . D$ is said to be compactly closed (open) in $X$ if $D \cap C$ is closed (open) in $C$ for each nonempty compact subset $C$ of $X$. A map $S: D \rightarrow 2^{Y}$ is said to be upper semi-continuous (u.s.c.) if for each $x \in D$ and for each open subset $U$ of $Y$ with $S(x) \subset U$, there exists an open neighborhood $V$ of $x$ in $X$ such that for each $z \in D \cap V, S(z) \subset U$. S is said to be compactly valued if for each $x \in D, S(x)$ is compact in $Y$.

The following notions which were introduced by Bardaro-Ceppitelli in [2] were motivated by an earlier work of Horvath [15].

A pair $\left(X,\left\{F_{A}\right\}\right)$ is called an $H$-space if $X$ is a topological space (which need not be Hausdorff) and $\left\{F_{A}\right\}$ is a family of nonempty contractible subsets of $X$ indexed by $A \in \mathscr{F}(X)$ such that $F_{A} \subset F_{A}$, whenever $A \subset A^{\prime}$. A subset $D$ of $X$ is said to be (i) $H$-convex if $F_{A} \subset D$ for each $A \in \mathscr{F}(D)$; (ii) weakly $H$-convex if $F_{A} \cap D$ is contractible for each $A \in \mathscr{F}(D)$ (this is equivalent to saying that $\left(D,\left\{F_{A} \cap D\right\}\right)$ is an $H$-space); (iii) $H$-compact in $X$ if, for each $A \in \mathscr{F}(X)$, there exists a compact, weakly $H$-convex subset $D_{A}$ of $X$ such that $D \cup A \subset D_{A}$. A map $F: X \rightarrow 2^{X}$ is called $H-K K M$ if $F_{A} \subset \bigcup_{x \in A} F(x)$ for each $A \in \mathscr{F}(X)$.

\section{Selection theorem, $H-K K M$ theorem and coincidence theorem}

The proof of the following useful result is contained in the proof of [15, Theorem 1] (see also [9]). 
LemMa 3.1. Let $X$ be a topological space. For each nonempty subset $J$ of $\{0, \ldots, n\}$, let $F_{J}$ be a nonempty contractible subset of $X$. If $J \subset J^{\prime}$ imply $F_{J} \subset F_{J^{\prime}}$, then there exists a continuous map $f: \Delta_{n} \rightarrow X$ such that $f\left(\Delta_{J}\right) \subset F_{J}$ for each nonempty subset $J$ of $\{0, \ldots, n\}$.

The following lemma is a slight improvement of [15, Corollary I.1] (also see [8]).

LeMMA 3.2. Let $\left(Y,\left\{F_{A}\right\}\right)$ be an $H$-space, $X$ be a nonempty subset of $Y$ and $G: X \rightarrow 2^{Y}$ be such that

(a) $G$ is an $H-K K M$ map;

(b) for each $x \in X, G(x)$ is closed and for some $x_{0} \in X, S\left(x_{0}\right)$ is compact.

Then $\bigcap_{x \in X} G(x) \neq \varnothing$.

THEOREM 3.1. Let $X$ be a compact topological space and $\left(Y,\left\{F_{A}\right\}\right)$ be an $H$-space. Suppose that $S, T: X \rightarrow 2^{Y}$ are such that

(a) for each $x \in X, S(x) \neq \varnothing$ and $F_{A} \subset T(x)$ for each $A \in \mathscr{F}(S(x))$;

(b) for each $y \in Y, S^{-1}(y)=\{x \in X: y \in S(x)\}$ is open in $X$.

Then $T$ has a continuous selection $g: X \rightarrow Y$ and there exists a finite set $A \in \mathscr{F}(Y)$ such that $g(X) \subset F_{A}$.

Proof. By (a), we must have $X=\bigcup_{y \in Y} S^{-1}(y)$. From (b) and the compactness of $X$ it follows that there exists a finite set

$$
A=\left\{y_{0}, \ldots, y_{n}\right\} \in \mathscr{F}(Y)
$$

such that $X=\bigcup_{i=0}^{n} S^{-1}(y)$. For each nonempty subset $J$ of $\{0, \ldots, n\}$, we define $F_{J}=F_{\left\{y_{j}\right\}_{j \in J}}$. Since $\left(Y,\left\{F_{A}\right\}\right)$ is an $H$-space, $F_{J}$ is a contractible subset of $Y$ and $F_{J} \subset F_{J^{\prime}}$ whenever $J \subset J^{\prime}$. By Lemma 3.1, there is a continuous map $f: \Delta_{n} \rightarrow Y$ such that $f\left(\Delta_{J}\right) \subset F_{J}$ for each nonempty subset $J$ of $\{0, \ldots, n\}$. Let $\left\{\alpha_{i}\right\}_{i=0}^{n}$ be a continuous partition of unity subordinate to the open covering $\left\{S^{-1}\left(y_{i}\right)\right\}_{i=0}^{n}$. Define a map $\psi: X \rightarrow \Delta_{n}$ by

$$
\psi(x)=\sum_{i=0}^{n} \alpha_{i}(x) e_{i} .
$$

For each $x \in X$, let $J(x)=\left\{i \in\{0, \ldots, n\}: \alpha_{i}(x) \neq 0\right\}$, then we have $\psi(x) \in \Delta_{J(x)}$ so that

$$
f \circ \psi(x) \in f\left(\Delta_{J(x)}\right) \subset F_{J(x)} \subset F_{A} .
$$


Since $x \in S^{-1}\left(y_{j}\right)$ for each $j \in J(x)$, it follows that $y_{j} \in S(x)$ for all $j \in J(x)$. By (a), we obtain $F_{J(x)} \subset T(x)$ so that $f \circ \psi(x) \in T(x)$ for each $x \in X$. Hence $g=f \circ \psi$ is a continuous selection of $T$ and there exists a finite set $A \in \mathscr{F}(Y)$ such that $g(X) \subset F_{A}$.

It would be of some interest to compare Theorem 3.1 with [15, Theorem 3].

Now we shall prove the following $H-K K M$ theorem.

THEOREM 3.2. Let $X$ be a nonempty subset of an $H$-space $\left(Y,\left\{F_{A}\right\}\right), Z$ be a topological space and $G: X \rightarrow 2^{Z}$ be such that

(a) for each $x \in X, G(x)$ is compactly closed in $Z$;

(b) there exists a compactly valued u.s.c. map $S: Y \rightarrow 2^{Z}$ such that the map $F: X \rightarrow 2^{Y}$ defined by $F(x)=S^{-1}(G(x))$ is $H-K K M$;

(c) there exists an $H$-compact subset $L$ of $Y$ and a nonempty compact subset of $Z$ such that for each $B \in \mathscr{F}(X)$ and for each $z \in S\left(L_{B}\right) \backslash K$, there is an $x \in L_{B} \cap X$ such that $x \notin G(x) \cap S\left(L_{B}\right)$. Then $K \cap\left(\cap_{x \in X} G(x)\right) \neq \varnothing$.

Proof. For each $x \in X$, let $G_{1}(x)=G(x) \cap K$, then $G_{1}(x)$ is closed in $K$ by (a). We shall prove that the family $\left\{G_{1}(x): x \in X\right\}$ has the finite intersection property. Let $B \in \mathscr{F}(X)$ be arbitrary fixed; then by (c), $L_{B}$ is a compact, weakly $H$-convex subset of $Y$ with $L \cup B \subset L_{B}$ such that for each $z \in S\left(L_{B}\right) \backslash K$, there is an $x \in L_{B} \cap X$ satisfying $z \notin G(x) \cap S\left(L_{B}\right)$. Now we define the map $G_{2}: L_{B} \cap \rightarrow 2^{L_{B}}$ by

$$
G_{2}(x)=F(x) \cap L_{B}=S^{-1}(G(x)) \cap L_{B} .
$$

Then we have the following properties.

(1) By the weak $H$-convexity of $L_{B},\left(L_{B},\left\{F_{A} \cap L_{B}\right\}\right)$ is an $H$-space.

(2) For each $A \in \mathscr{F}\left(L_{B} \cap X\right) \subset \mathscr{F}(X)$, we have $F_{A} \subset \bigcup_{x \in A} F(x)$ by (b) so that $F_{A} \cap L_{B} \subset \bigcup_{A \in A}\left(F(x) \cap L_{B}\right)=\bigcup_{x \in A} G_{2}(x)$. Thus $G_{2}$ is also an $H-K K M$ map.

(3) Since $S$ is compactly valued u.s.c. and $L_{B}$ is compact in $Y$, it follows that $S\left(L_{B}\right)$ is compact in $Z$ so that for each $x \in X, G(x) \cap S\left(L_{B}\right)$ is closed in $Z$ by (a). By the upper semi-continuity of $S, S^{-1}\left(G(x) \cap S\left(L_{B}\right)\right)$ is a closed subset of $X$. Hence, for each $x \in L_{B} \cap X$,

$$
G_{2}(x)=S^{-1}(G(x)) \cap L_{B}=S^{-1}\left(G(x) \cap S\left(L_{B}\right)\right) \cap L_{B}
$$

is compact in $L_{B}$. 
By Lemma 3.2, $\bigcap_{x \in L_{B} \cap X} G_{2}(x) \neq \varnothing$. Take any $y \in \bigcap_{x \in L_{B} \cap X} G_{2}(x)$, then we have

$$
S(y) \cap\left(\bigcap_{x \in L_{B} \cap X}\left(G(x) \cap S\left(L_{B}\right)\right)\right) \neq \varnothing .
$$

By (c), we must have

$$
\begin{aligned}
S(y) & \cap\left(\bigcap_{x \in L_{B} \cap X}\left(G(x) \cap S\left(L_{B}\right)\right)\right) \subset S(y) \cap\left(\bigcap_{x \in L_{B} \cap X}(G(x) \cap K)\right) \\
& \subset S(y) \cap\left(\bigcap_{x \in B}(G(x) \cap K)\right)=S(y) \cap\left(\bigcap_{x \in B} G_{1}(x)\right) \subset \bigcap_{x \in B} G_{1}(x) .
\end{aligned}
$$

It follows that $\bigcap_{x \in B} G_{1}(x) \neq \varnothing$. By the compactness of $K, \bigcap_{x \in X} G_{1}(x) \neq$ $\varnothing$, that is, $K \cap\left(\bigcap_{x \in X} G(x)\right) \neq \varnothing$.

ReMark 3.1. If $S$ is a single-valued continuous map, Theorem 3.2 reduces to [10, Theorem 1] and in turn generalizes [1, Theorem 1]. It is easy to see that condition (c) of Theorem 3.2 is equivalent to the condition:

$\left(c_{1}\right)$ there exists an $H$-compact subset $L$ of $Y$ and a nonempty compact subset $K$ of $Z$ such that for each $B \in \mathscr{F}(X)$,

$$
\bigcap_{x \in L_{B} \cap X}\left(G(x) \cap S\left(L_{B}\right)\right) \subset K .
$$

We also note that under hypothesis (a) of Theorem 3.2, condition $\left(c_{1}\right)$ is implied by the condition: there exists an $H$-compact subset $L$ of $Y$ such that $\bigcap_{x \in L \cap X} G(x)$ is compact in $Z$. Since every convex space is an $H$-space [17], Theorem 3.2 generalizes [17, Theorem I] (which is equivalent to [19, Theorem 6] to an $H$-space with a weaker assumption.

In the following we shall prove a coincidence theorem.

Theorem 3.3. Let $X$ be a nonempty subset of an $H$-space $\left(Y,\left\{F_{A}\right\}\right), Z$ be a topological space and $A, B: X \rightarrow 2^{Z}$ be such that

(a) for each $z \in Z, B^{-1}(z) \neq \varnothing$ and $F_{D} \subset A^{-1}(z)$ for each $D \in$ $\mathscr{F}\left(B^{-1}(z)\right)$;

(b) for each $x \in X, B(x)$ is compactly open in $Z$;

(c) there exists an $H$-compact subset $L$ of $Y$ and a nonempty compact subset $K$ of $Z$ such that for each $B \in \mathscr{F}(X)$ and for each $z \in Z \backslash K$, there is an $x \in L_{B} \cap X$ such that $z \in B(x)$.

Then for any compactly valued u.s.c. map $S: Y \rightarrow 2^{Z}$, there exists an $x_{0} \in X$ such that $S\left(x_{0}\right) \subset A\left(x_{0}\right)$. 
Proof. Define a map $G: X \rightarrow 2^{X}$ by

$$
G(x)=Z \backslash B(x) \quad \text { for each } x \in X .
$$

Then we have the following properties.

(1) For each $x \in X, G(x)$ is compactly closed by (b).

(2) By (c), there exist an $H$-compact subset $L$ of $Y$ and a nonempty compact subset $K$ of $Z$ such that for each $B \in \mathscr{F}(X)$ and for each $z \in$ $Z \backslash K$, there is an $x \in L_{B} \cap X$ such that $z \notin G(x)$ so that $z \notin G(x) \cap S\left(L_{B}\right)$ for any compactly valued u.s.c. map $S: Y \rightarrow 2^{Z}$.

Now for any given compactly valued u.s.c. map $S: Y \rightarrow 2^{Z}$, define a map $F: X \rightarrow 2^{Y}$ by

$$
F(x)=S^{-1}(G(x)) \quad \text { for each } x \in X .
$$

If $F$ is an $H-K K M$ map, it follows from Theorem 3.2 that

$$
\bigcap_{x \in X} G(x)=\bigcap_{x \in X}(Z \backslash B(x))=Z / \bigcup_{x \in X} B(x) \neq \varnothing .
$$

But condition (a) implies $Z=\bigcup_{x \in X} B(x)$, we obtain a contradiction so that $F$ is not an $H-K K M$ map. Therefore there exists $D \in \mathscr{F}(X)$ and $x_{0} \in F_{D}$ such that $x_{0} \notin \bigcup_{x \in D} F(x)=\bigcup_{x \in D} S^{-1}(G(x))$. It follows that $S\left(x_{0}\right) \cap\left(\bigcup_{x \in D} G(x)\right)=S\left(x_{0}\right) \cap\left(\bigcup_{x \in D}(Z \backslash B(x))=\varnothing\right.$. Thus, $S\left(x_{0}\right) \subset B(x)$ for all $x \in D$ so that for any given $z \in S\left(x_{0}\right)$, we have $D \in \mathscr{F}\left(B^{-1}(z)\right)$. By (a), $F_{D} \subset A^{-1}(z)$. It follows that $x_{0} \in A^{-1}(z)$ and so $z \in A\left(x_{0}\right)$. From the arbitrariness of $z \in S\left(x_{0}\right)$ it follows that $S\left(x_{0}\right) \subset A\left(x_{0}\right)$.

REMARK 3.2. We note that condition (c) of Theorem 3.3 is equivalent to the following condition:

(c') there exists an $H$-compact subset $L$ of $Y$ and a compact subset $K$ of $Z$ such that

$$
Z \backslash \bigcup_{x \in L_{B} \cap X} B(x) \subset K
$$

Theorem 3.3 improves and generalizes [4, Theorem 1, 6, Theorem 1, 16, Theorem 3.1, 17, Theorem 1.1, 19, Theorem 6, 20, Theorem 4.3 and 23, Theorem 2 and 5].

\section{Intersection theorems concerning sets with $H$-convex sections}

In this section, we always assume that every $H$-space $\left(X,\left\{F_{A}\right\}\right)$ has the following property: for each $A \in \mathscr{F}(X), F_{A}$ is $H$-compact in $X$. Clearly, 
each convex space $X$ is an $H$-space [17] with the property that $F_{A}=\operatorname{co}(A)$ for each $A \in \mathscr{F}(X)$.

The following notations are used throughout this section. Let $\left(X_{i},\left\{F_{A_{i}}\right\}\right)$, $i=1, \ldots, n$, be $n(\geq 2) H$-spaces and $X=\prod_{i=1}^{n} X_{i}$. For each $i \in$ $\{1, \ldots, n\}$, let $\widehat{X}_{i}=\prod_{j \neq i} X_{j}$. Also $\hat{x}_{i}$ denotes an element of $\widehat{X}_{i}$. For each $i=1, \ldots, n, X_{i} \times \widehat{X}_{i}=X$ and $\left(x_{i}, \hat{x}_{i}\right)$ denotes an element of $X$ (with the appropriate ordering).

We shall prove the following intersection theorems.

TheOREM 4.1. Let $\left(X_{i},\left\{F_{A_{i}}\right\}\right), i=1, \ldots, n$, be $n$ ( $\left.\geq 2\right) H$-spaces and $X=\prod_{i=1}^{n} X_{i}$. If $M_{1}, \ldots, M_{n}, N_{1}, \ldots, N_{n}$ are $2 n$ subsets of $X$ such that

(a) for each $i \in\{1, \ldots, n\}$ and for each $x_{i} \in X_{i}$, the section $M_{i}\left(x_{i}\right)=$ $\left\{\hat{y}_{i} \in \widehat{X}_{i}:\left(x_{i}, \hat{y}_{i}\right) \in M_{i}\right\}$ is compactly open in $\widehat{X}_{i}$;

(b) for each $i \in\{1, \ldots, n\}$ and for each $\hat{y}_{i} \in \hat{X}_{i}$, the section $M_{i}\left(\hat{y}_{i}\right)=$ $\left\{x_{i} \in X_{i}:\left(x_{i}, \hat{y}_{i}\right) \in M_{i}\right\} \neq \varnothing$ and $F_{D_{i}} \subset N_{i}\left(\hat{y}_{i}\right)=\left\{x_{i} \in X_{i}:\left(x_{i}, \hat{y}_{i}\right) \in N_{i}\right\}$ for each $D_{i} \in \mathscr{F}\left(M_{i}\left(\hat{y}_{i}\right)\right)$;

(c) for at least $(n-1)$ indices $i$, there exists an $H$-compact subset $L_{i}$ of $X_{i}$ such that $\widehat{X}_{i} \backslash \bigcup_{x_{i} \in L_{i}} M_{i}\left(x_{i}\right)$ is compact in $\widehat{X}_{i}$. Then $\bigcap_{i=1}^{n} N_{i} \neq \varnothing$.

Proof. We may assume without loss of generality that condition (c) holds for $i=2, \ldots, n$. By (b), we have

$$
\widehat{X}_{i}=\bigcup_{x_{i} \in X_{i}} M\left(x_{i}\right) \text { for each } i=1, \ldots, n .
$$

From (a), (c) and (4.1) it follows that for each $i=2, \ldots, n$, there exists a finite set $B_{i}=\left\{x_{i}^{1}, \ldots, x_{i}^{k_{i}}\right\} \in \mathscr{F}\left(X_{i}\right)$ such that

$$
\widehat{X}_{i} \backslash \bigcup_{x_{i} \in L_{i}} M_{i}\left(x_{i}\right) \subset \bigcup_{j=1}^{k_{i}} M_{i}\left(x_{i}^{j}\right) .
$$

Thus, we have

$$
\widehat{X}_{i} \subset \bigcup_{x_{i} \in L_{i} \cup\left\{x_{i}^{1}, \ldots, x_{i}^{k_{i}}\right\}} M_{i}\left(x_{i}\right) .
$$

Since $L_{i}$ is $H$-compact in $X_{i}$, there exists a compact, weakly $H$-convex subset $C_{i}$ of $X_{i}$ with $L_{i} \cup B_{i} \subset C_{i}$ and (4.2) imply

$$
\widehat{X}_{i} \subset \bigcup_{x_{i} \in C_{i}} M_{i}\left(x_{i}\right) \text {. }
$$


Now we define the maps $M_{1}, N_{1}: \prod_{i=2}^{n} C_{i} \rightarrow 2^{X_{1}}$ as follows: for each $\hat{y}_{1} \in \prod_{i=2}^{n} C_{i}$,

$$
M_{1}\left(\hat{y}_{1}\right)=\left\{x_{1} \in X_{1}:\left(x_{1}, \hat{y}_{1}\right) \in M_{1}\right\}
$$

and

$$
N_{1}\left(\hat{y}_{1}\right)=\left\{x_{1} \in X_{1}:\left(x_{1}, \hat{y}_{1}\right) \in N_{1}\right\} .
$$

By (b), for each $\hat{y}_{1} \in \prod_{i=2}^{n} C_{i}, M_{1}\left(\hat{y}_{1}\right) \neq \varnothing$ and $F_{D_{1}} \subset N_{1}\left(\hat{y}_{1}\right)$ for each $D_{1} \in \mathscr{F}\left(M_{1}\left(\hat{y}_{1}\right)\right)$. For each $x_{1} \in X_{1}$,

$$
M_{1}^{-1}\left(x_{1}\right)=\left\{\hat{y}_{1} \in \prod_{i=2}^{n} C_{i}:\left(x_{1}, \hat{y}_{1}\right) \in M_{1}\right\}=\prod_{i=2}^{n} C_{i} \cap M_{1}\left(x_{1}\right)
$$

is open in $\prod_{i=2}^{n} C_{i}$ by (a). It follows from Theorem 3.1 that there is a continuous map $g: \prod_{i=2}^{n} C_{i} \rightarrow X_{1}$ and $A_{1} \in \mathscr{F}\left(X_{1}\right)$ such that $g\left(\hat{y}_{1}\right) \in$ $N_{1}\left(\hat{y}_{1}\right)$ for each $y_{1} \in \prod_{i=2}^{n} C_{i}$ and $g\left(\prod_{i=2}^{n} C_{i}\right) \subset F_{A_{1}}$. By the assumption that $F_{A_{1}}$ is $H$-compact, there exists a compact weakly $H$-convex subset $C_{1}$ of $X_{1}$ with $F_{A_{1}} \subset C_{1}$. Hence, we have $g\left(\prod_{i=2}^{n} C_{i}\right) \subset C_{1}$ and $\left(g\left(\hat{y}_{1}\right), \hat{y}_{1}\right) \in N_{1}$ for each $\hat{y}_{1} \in \prod_{i=2}^{n} C_{i}$.

Let $C=\prod_{i=1}^{n} C_{i}$ and $\widehat{C}_{i}=\prod_{j \neq i} C_{j}$. For each $i \in\{2, \ldots, n\}$, we define the maps $M_{i}, N_{i}: C_{i} \rightarrow 2^{\widehat{C}_{i}}$ by

$$
M_{i}\left(x_{i}\right)=\left\{\hat{y}_{i} \in \widehat{C}_{i}:\left(x_{i}, \hat{y}_{i}\right) \in M_{i}\right\}
$$

and

$$
N_{i}\left(x_{i}\right)=\left\{\hat{y}_{i} \in \widehat{C}_{i}:\left(x_{i}, \hat{y}_{i}\right) \in N_{i}\right\}
$$

for each $x_{i} \in C_{i}$. Then, for each $x_{i} \in C_{i}, M_{i}\left(x_{i}\right)$ is open in $\hat{C}_{i}$ by (a) and for each $\hat{y}_{i} \in \widehat{C}_{i}, M_{i}^{-1}\left(\hat{y}_{i}\right)=\left\{x_{i} \in C_{i}:\left(x_{i}, \hat{y}_{i}\right) \in M_{i}\right\}=C_{i} \cap M_{i}\left(\hat{y}_{i}\right) \neq \varnothing$ and $F_{D_{i}} \subset N_{i}^{-1}\left(\hat{y}_{i}\right)$ for each $D_{i} \in \mathscr{F}\left(M_{i}^{-1}\left(\hat{y}_{i}\right)\right)$ by (b) and (4.3). From Theorem 3.3 with $X=Y=C_{i}$ and $Z=\widehat{C}_{i}=K$ it follows that for any compactly valued u.s.c. map $S: C_{i} \rightarrow 2^{\widehat{C}_{i}}$ there is an $x_{i} \in C_{i}$ such that $S\left(x_{i}\right) \subset N_{i}\left(x_{i}\right)$.

Now, let $p_{i}: \widehat{C}_{1} \rightarrow C_{i}, i=2, \ldots, n$ and $q_{i}: \widehat{C}_{i} \rightarrow C_{1}, i=1, \ldots, n$ be the projective maps, then $p_{i}, q_{i}$ are continuous open maps. We consider the following map

$$
q_{i}^{-1} \circ g \circ p_{i}^{-1}: C_{i} \rightarrow 2^{\widehat{C}_{i}}, \quad i=2, \ldots, n .
$$

Since $p_{i}$ and $q_{i}$ are continuous open maps and $g$ is continuous, it is easy to see that $q_{i}^{-1} \circ g \circ p_{i}^{-1}$ is compactly valued and u.s.c. on $C_{i}$. Thus for $i=2, \ldots, n$, there exists $x_{i} \in C_{i}$ such that

$$
q_{i}^{-1} \circ g \circ p_{i}^{-1}\left(x_{i}\right) \subset N_{i}\left(x_{i}\right) \text {. }
$$


Let $\hat{x}_{1}=\left(x_{2}, \ldots, x_{n}\right)$ and $g\left(\hat{x}_{1}\right)=x_{1}$, then

$$
x=\left(x_{1}, \ldots, x_{n}\right) \in N_{1} \text {. }
$$

Since, for $i=2, \ldots, n$,

$$
x_{1}=g\left(\hat{x}_{1}\right) \in g\left(C_{2} \times \cdots \times C_{i-1} \times\left\{x_{i}\right\} \times C_{i} \times \cdots \times C_{n}\right)
$$

and

$$
\begin{aligned}
q_{i}^{-1} \circ g \circ p_{i}^{-1}= & g\left(C_{2} \times \cdots \times C_{i-1} \times\left\{x_{i}\right\} \times C_{i} \times \cdots \times C_{n}\right) \\
& \times C_{2} \times \cdots \times C_{i-1} \times C_{1} \times \cdots \times C_{n},
\end{aligned}
$$

we must have

$$
\hat{x}_{i}=\prod_{j \neq i} x_{i} \in q_{i}^{-1} \circ g \circ p_{i}^{-1}\left(x_{i}\right) \subset N_{i}\left(x_{i}\right) \text { for } i=2, \ldots, n .
$$

Hence $x=\left(x_{1}, \ldots, x_{n}\right) \in N_{i}$ for all $i=1, \ldots, n$ so that $\prod_{i=1}^{n} N_{i} \neq \varnothing$.

REMARK 4.1. Theorem 4.1 generalizes [17, Theorem 1.9] to $2 n$ sets and $H$-spaces with weaker assumptions. We observe that condition (c) of Theorem 4.1 is implied by the following condition:

$\left(c_{1}\right)$ at least $(n-1)$ of the $X_{i}$ 's (say $\left.X_{2}, \ldots, X_{n}\right)$ are compact. Indeed, in the case, (c) is satisfied by $L_{i}=X_{i}$ for $i=2, \ldots, n$, because by (b) the set $\widehat{X}_{i} \backslash \cup_{x_{i} \in X_{i}} M_{i}\left(x_{i}\right)=\varnothing$. Thus Theorem 4.1 also generalizes [11, Theorem 1] to $H$-spaces. It would be of some interest to compare Theorem 4.1 with [3, Theorem 2].

TheOREM 4.2. Let $\left(X_{i},\left\{F_{A_{i}}\right\}\right), i=1, \ldots, n$, be $(\geq 2) H$-spaces and $X=\prod_{i=1}^{n} X_{i}$. If $M_{1}, \ldots, M_{n}, N_{1}, \ldots, N_{n}$ are $2 n$ subsets of $X$ such that

(a) for each $i \in\{1, \ldots, n\}$ and for each $x_{i} \in X_{i}$, the section $M_{i}\left(x_{i}\right)$ is compactly open in $\widehat{X}_{i}$;

(b) for each $i \in\{1, \ldots, n\}$ and for each $\hat{y}_{i} \in \hat{X}_{i}$, the section $M_{i}\left(\hat{y}_{i}\right) \neq \varnothing$ and $F_{D_{i}} \subset N_{i}\left(\hat{y}_{i}\right)$ for each $D_{i} \in \mathscr{F}\left(M_{i}\left(\hat{y}_{i}\right)\right)$;

(c) for at least $(n-1)$ indices $i$, there exists an $H$-compact subset $L_{i}$ of $X_{i}$ and a compact subset $\widehat{K}_{i}$ of $\widehat{X}_{i}$ such that $L_{i} \cap M_{i}\left(\hat{y}_{i}\right) \neq \varnothing$ for each $\hat{y}_{i} \in \widehat{X}_{i} \backslash \widehat{K}_{i}$.

Then $\bigcap_{i=1}^{n} N_{i} \neq \varnothing$.

Proof. We shall show that condition (c) is equivalent to condition (c) of Theorem 4.1 and hence Theorem 4.2 follows from Theorem 4.1. Suppose that condition (c) of Theorem 4.1 holds. Let $\widehat{X}_{i} \backslash \bigcup_{x_{i} \in L_{i}} M\left(x_{i}\right)=\widehat{K}_{i}$, then $\widehat{K}_{i}$ is a compact subset of $\widehat{X}_{i}$ and for each $\hat{y}_{i} \in \widehat{X}_{i} \backslash \widehat{K}_{i}, \hat{y}_{i} \in \bigcup_{x_{i} \in L_{i}} M_{i}\left(x_{i}\right)$. 
Thus, there exists $x_{i} \in L_{i}$ such that $\left(x_{i}, \hat{y}_{i}\right) \in M_{i}$, that is $x_{i} \in L_{i} \cap M_{i}\left(\hat{y}_{i}\right)$ and hence $L_{i} \cap M_{i}\left(\hat{y}_{i}\right) \neq \varnothing$. Therefore condition (c) of Theorem 4.2 holds. If condition (c) of Theorem 4.2 holds, then for each $\hat{y}_{i} \in \widehat{X}_{i} \backslash \bigcup_{x_{i} \in L_{i}} M_{i}\left(x_{i}\right)$, $\hat{y}_{i} \notin M_{i}\left(x_{i}\right)$ for all $x_{i} \in L_{i}$ so that $x_{i} \notin M_{i}\left(\hat{y}_{i}\right)$ for all $x_{i} \in L_{i}$. Thus $L_{i} \cap M_{i}\left(\hat{y}_{i}\right)=\varnothing$. It follows that $\hat{y}_{i} \in \widehat{K}_{i}$ and

$$
\widehat{X}_{i} \backslash \bigcup_{x_{i} \in L_{i}} M_{i}\left(x_{i}\right) \subset \widehat{K}_{i} .
$$

By (a), $\widehat{X}_{i} \backslash \bigcup_{x_{i} \in L_{i}} M_{i}\left(x_{i}\right)$ is closed in $\widehat{K}_{i}$ so that it is compact in $\widehat{X}_{i}$. This proves that condition (c) of Theorem 4.1 holds.

THEOREM 4.3. Let $\left(X_{i},\left\{F_{A_{i}}\right\}\right), i=1, \ldots, n$, be $n(\geq 2) H$-spaces and $X=\prod_{i=1}^{n} X_{i}$. If $M_{1}, \ldots, M_{n}, N_{1}, \ldots, N_{n}$ are $2 n$ subsets of $X$ such that

(a) for each $i \in\{1, \ldots, n\}$ and for each $x_{i} \in X_{i}$, the section $M_{i}\left(x_{i}\right)$ is compactly open in $\hat{X}_{i}$;

(b) for each $i \in\{1, \ldots, n\}$ and for each $\hat{y}_{i} \in \widehat{X}_{i}$, the section $M_{i}\left(\hat{y}_{i}\right) \neq \varnothing$ and $F_{D_{i}} \subset N_{i}\left(\hat{y}_{i}\right)$ for each $D_{i} \in \mathscr{F}\left(M_{i}\left(\hat{y}_{i}\right)\right)$;

(c) there exists a compact subset $K$ of $X$ such that for each $i=1, \ldots, n$, the projection $L_{i}$ of $K$ on $X_{i}$ is $H$-compact in $X_{i}$ and such that $K \cap$ $\left(\prod_{i=1}^{n} M_{i}\left(y_{i}\right)\right) \neq \varnothing$ for each $y \in X \backslash K$.

Then $\bigcap_{i=1}^{n} N_{i} \neq \varnothing$.

Proof. For each $i=1, \ldots, n$, let $L_{i}$ and $\widehat{K}_{i}$ be the projections of $K$ on $X_{i}$ and $\hat{X}_{i}$, respectively, then $L_{i}$ is $H$-compact in $X_{i}$ by the assumption and $\widehat{K}_{i}$ is a compact subset of $\widehat{X}_{i}$. The condition (c) of Theorem $4.3 \mathrm{imply}$ that for each $i=1, \ldots, n, L_{i} \cap M_{i}\left(\hat{y}_{i}\right) \neq \varnothing$ for each $\hat{y}_{i} \in \hat{X}_{i} \backslash \widehat{K}_{i}$. By Theorem 4.2, $\bigcap_{i=1}^{n} N_{i} \neq \varnothing$.

Remark 4.3. Theorem 4.3 generalizes [12, Theorem 11] in several ways. We note that if condition (b) of Theorem 4.3 is replaced by the following condition:

$\left(\mathrm{b}_{1}\right)$ for each $i \in\{1, \ldots, n\}$ and for each $y_{i} \in X_{i}$, the section $M_{i}\left(y_{i}\right) \neq \varnothing$ and for at least $q(\geq 2)$ indices $i, F_{D_{i}} \subset N_{i}\left(\hat{y}_{i}\right)$ for each $D_{i} \in \mathscr{F}\left(M_{i}\left(\hat{y}_{i}\right)\right)$ and for each $\hat{y}_{i} \in \widehat{X}_{i}$.

Then at least $q$ of the sets $N_{1}, \ldots, N_{n}$ have a nonempty intersection by applying Theorem 4.3 for the $q H$-spaces satisfying condition $\left(\mathrm{b}_{1}\right)$. Thus Theorem 4.3 also generalizes [13, Theorem 15]. 


\section{Some applications to the von Neumann Minimax Theorem}

For convenience, we state the special case $n=2$ of Theorem 4.1.

Theorem 5.1. Let $\left(X,\left\{F_{A}\right\}\right)$ and $\left(Y,\left\{F_{A}\right\}\right)$ be two $H$-spaces and let $M_{1}, M_{2}, N_{1}, N_{2}$ be subsets of $X \times Y$. Suppose that

(a) for each $x \in X$, the section $M_{1}(x)=\left\{y \in Y:(x, y) \in M_{1}\right\}$ is compactly open in $Y$, the section $M_{2}(x)=\left\{y \in Y:(x, y) \in M_{2}\right\} \neq \varnothing$ and $F_{A} \subset N_{2}(x)$ for each $A \in \mathscr{F}\left(M_{2}(x)\right)$;

(b) for each $y \in Y$, the section $M_{2}(y)=\left\{x \in X:(x, y) \in M_{2}\right\}$ is compactly open in $X$, the section $M_{1}(y)=\left\{x \in X:(x, y) \in M_{1}\right\} \neq \varnothing$ and $F_{A} \subset N_{1}(y)$ for each $A \in \mathscr{F}\left(M_{1}(y)\right)$;

(c) there exists an $H$-compact subset $X_{0}$ of $X$ such that the intersection $\bigcap_{x \in X_{0}}\left(Y \backslash M_{1}(x)\right)$ is compact in $Y$.

Then the intersection $N_{1} \cap N_{2}$ is nonempty.

REMARK 5.1. If the coercive condition (c) is replaced by the following condition:

$\left(\mathrm{c}_{1}\right)$ there exists an $H$-compact subset $Y_{0}$ of $Y$ such that the intersection $\bigcap_{y \in Y_{0}}\left(X \backslash M_{2}(y)\right)$ is compact in $X$, then the inclusion of Theorem 5.1 still holds. We also note that if at least one of $X$ or $Y$ is compact, then condition (c) of Theorem 5.1 holds. Theorem 5.1 improves and generalizes [22, Theorem 2] and Ha's result [14] in several ways.

TheOREM 5.2. Let $\left(X,\left\{F_{A}\right\}\right)$ and $\left(Y,\left\{F_{A}\right\}\right)$ be two $H$-spaces and $f, s, t, g: X \times Y \rightarrow \mathbb{R}$ and $\lambda \in \mathbb{R}$ be such that

(a) $s \leq t$ on $X \times Y$;

(b) for each $x \in X, y \mapsto f(x, y)$ is lower semi-continuous on each compact subset of $Y$ and for each $y \in Y, x \mapsto g(x, y)$ is upper semi-continuous on each compact subset of $X$;

(c) for each $x \in X, A \in \mathscr{F}(\{y \in Y: g(x, y)<\lambda\})$ imply $F_{A} \subset\{y \in Y$ : $t(x, y)<\lambda\}$ and for each $y \in Y, A \in \mathscr{F}(\{x \in X: f(x, y)>\lambda\})$ imply $F_{A} \subset\{x \in X: s(x, y)>\lambda\}$;

(d) there exists an $H$-compact subset $X_{0}$ of $X$ such that the intersection $\bigcap_{x \in X_{0}}(Y \backslash\{y \in Y: f(x, y)>\lambda\})$ is compact in $Y$.

Then either there exists $\hat{y} \in Y$ such that $f(x, \hat{y}) \leq \lambda$ for all $x \in X$ or there exists $\hat{x} \in X$ such that $g(\hat{x}, y) \geq \lambda$ for all $y \in \bar{Y}$. 
Proof. Suppose that the conclusion does not hold. Let

$$
\begin{aligned}
& M_{1}=\{(x, y) \in X \times Y: f(x, y)>\lambda\}, \\
& \left.M_{2}=\{x, y) \in X \times Y: g(x, y)<\lambda\right\}, \\
& N_{1}=\{(x, y) \in X \times Y: s(x, y)>\lambda\}, \\
& N_{2}=\{(x, y) \in X \times Y: t(x, y)<\lambda\} .
\end{aligned}
$$

Then for each $x \in X$,

$$
M_{2}(x)=\{y \in Y: g(x, y)<\lambda\} \neq \varnothing
$$

and for each $y \in Y$,

$$
M_{1}(y)=\{x \in X: f(x, y)>\lambda\} \neq \varnothing .
$$

Moreover,

(i) for each $x \in X, M_{1}(x)=\{y \in Y: f(x, y)>\lambda\}$ is compactly open in $Y$ and for each $y \in Y, M_{2}(y)=\{x \in X: g(x, y)<\lambda\}$ is compactly open in $X$ by (a);

(ii) for each $x \in X, F_{A} \subset N_{2}(x)$ whenever $A \in \mathscr{F}\left(M_{2}(x)\right)$ and for each $y \in Y, F_{A} \subset N_{1}(y)$ whenever $A \in \mathscr{F}\left(M_{1}(y)\right)$ by (c);

(iii) condition (c) of Theorem 5.1 holds by (d).

Thus all hypotheses of Theorem 5.1 are satisfied so that $N_{1} \cap N_{2} \neq \varnothing$. Take any $(\hat{x}, \hat{y}) \in N_{1} \cap N_{2}$, then $s(\hat{x}, \hat{y})>\lambda$ which contradicts (a). Therefore the conclusion must hold.

Recall that a real-valued function $\varphi$ defined on an $H$-space $\left(X,\left\{F_{A}\right\}\right)$ is said to be $H$-quasi-concave if for each real number $t$, the set $\{x \in X$ : $\varphi(x)>t\}$ is $H$-convex; $\varphi$ is said to be $H$-quasi-convex if $-\varphi$ is $H$-quasiconcave.

Corollary 5.1. Let $\left(X,\left\{F_{A}\right\}\right)$ and $\left(Y,\left\{F_{A}\right\}\right)$ be two $H$-spaces and $f, s, t, g: X \times Y \rightarrow \mathbb{R}$ be such that

(a) $f \leq s \leq t \leq g$ on $X \times Y$;

(b) for each $x \in X, y \mapsto f(x, y)$ is lower semi-continuous on each compact subset of $Y$ and for each $y \in Y, x \mapsto g(x, y)$ is upper semi-continuous on each compact subset of $X$;

(c) for each $x \in X, t(x, y)$ is an $H$-quasi-convex function of $y$ on $Y$ and for each $y \in Y, s(x, y)$ is an $H$-quasi-concave function of $x$ on $X$;

(d) there exists an $H$-compact subset $X_{0}$ of $X$ such that for each $t \in \mathbb{R}$, the intersection $\bigcap_{x \in X_{0}}(Y \backslash\{y \in Y: f(x, y)>t\})$ is compact in $Y$.

Then for each $\lambda \in \mathbb{R}$, either there exists $\hat{y} \in Y$ such that $f(x, \hat{y}) \leq \lambda$ for all $x \in X$ or there exists $\hat{x} \in X$ such that $g(\hat{x}, y) \geq \lambda$ for all $y \in Y$. 
REMARK 5.2. Theorem 5.2 and Corollary 5.1 improve and generalize [ 5 , Theorem 5.4]. It would be of some interest to compare Theorem 5.2 and Corollary 5.1 with [8, Theorem 4 and Corollary 4$]$.

TheOREM 5.3. Let $\left(X,\left\{F_{A}\right\}\right)$ and $\left(Y,\left\{F_{A}\right\}\right)$ be two H-spaces and $f, s, t, g: Y \times Y \rightarrow \mathbb{R}$ be such that

(a) $s \leq t$ on $X \times Y$;

(b) for each $x \in X, y \mapsto f(x, y)$ is lower semi-continuous on each compact subset of $Y$ and for each $y \in Y, x \mapsto g(x, y)$ is upper semi-continuous on each compact subset of $X$;

(c) for each $\gamma \in \mathbb{R}$ and for each $x \in X, F_{A} \subset\{y \in Y: t(x, y)<\gamma\}$ whenever $A \in \mathscr{F}(\{y \in Y: g(x, y)<\gamma)$, and for each $\gamma \in \mathbb{R}$ and for each $y \in Y, F_{A} \subset\{x \in X: s(x, y)>\gamma\}$ whenever $A \in \mathscr{F}(\{x \in X: f(x, y)>$ $\gamma\})$;

(d) there exists an $H$-compact subset $L$ of $X$ and a compact subset $K$ of $Y$ such that

$$
\inf _{y \in Y} \sup _{x \in X} f(x, y) \leq \inf _{y \in Y \backslash K} \sup _{x \in L} f(x, y) .
$$

Then the following minimax inequality holds,

$$
\alpha=\inf _{y \in Y} \sup _{x \in X} f(x, y) \leq \sup _{x \in X} \inf _{y \in Y} g(x, y)=\beta .
$$

Proof. Without loss of generality, we may assume that $\alpha \neq-\infty$ and $\beta \neq+\infty$. Assume to the contrary that $\alpha>\beta$. Choose a real number $\lambda$ such that $\alpha>\lambda>\beta$. Let

$$
\begin{aligned}
M_{1} & =\{(x, y) \in X \times Y: f(x, y)>\lambda\}, \\
M_{2} & =\{(x, y) \in X \times Y: g(x, y)<\lambda\}, \\
N_{1} & =\{(x, y) \in X \times Y: s(x, y)>\lambda\}, \\
N_{2} & =\{(x, y) \in X \times Y: t(x, y)<\lambda\} .
\end{aligned}
$$

Then $\alpha>\lambda$ implies that for each $y \in Y, M_{1}(y) \neq \varnothing$; and $\lambda>\beta$ implies that for each $x \in X, M_{2}(x) \neq \varnothing$. The condition (d) implies that $\bigcap_{x \in L}\left(Y \backslash M_{1}(x)\right) \subset K$ and each $M_{1}(x)$ is compactly open in $Y$ by (b), thus $\bigcap_{x \in L}\left(Y \backslash M_{1}(x)\right)$ is compact in $Y$. The other conditions of Theorem 5.1 are easily verified. By Theorem 5.1, $N_{1} \cap N_{2} \neq \varnothing$ so that there exists $(\hat{x}, \hat{y}) \in X \times Y$ such that $s(\hat{x}, \hat{y})>\lambda$ and $t(\hat{x}, \hat{y})<\lambda$ which contradicts (a). This completes the proof.

Corollary 5.2. Let $\left(X,\left\{F_{A}\right\}\right)$ an $d\left(Y,\left\{F_{A}\right\}\right)$ be two- $H$-spaces and $f, s, t, g: X \times Y \rightarrow \mathbb{R}$ be such that

(a) $f \leq s \leq t \leq g$ on $X \times Y$; 
(b) for each $x \in X, y \mapsto f(x, y)$ is lower semi-continuous on each compact subset of $Y$ and for each $y \in Y, x \mapsto g(x, y)$ is upper semi-continuous on each compact subset of $X$;

(c) for each $x \in X, t(x, y)$ is an $H$-quasi-convex function of $y$ on $Y$ for each $y \in Y, s(x, y)$ is an $H$-quasi-concave function of $x$ on $X$;

(d) there exists an $H$-compact subset $L$ of $X$ and a compact subset $K$ of $Y$ such that

$$
\inf _{y \in Y} \sup _{x \in X} f(x, y) \leq \inf _{y \in Y \backslash Y} \sup _{x \in L} f(x, y) .
$$

Then the following minimax inequality holds,

$$
\alpha=\inf _{y \in Y} \sup _{x \in X} f(x, y) \leq \sup _{x \in X} \inf _{y \in Y} g(x, y)=\beta .
$$

REMARK 5.3. Theorem 5.3 and Corollary 5.2 generalizes [22, Theorem $4(2), 3$, Corollary 5.5] and Liu's result [18] in several ways. When $f=s=$ $t=g$, the conclusion of Corollary 5.2 (respectively Theorem 5.3) implies the following minimax equality, which generalizes the minimax principle of the von Neumann type due to Sion [21]:

$$
\inf _{y \in Y} \sup _{x \in X} f(x, y)=\sup _{x \in X} \inf _{y \in Y} f(x, y) .
$$

It would be of some interest to compare the minimax equality with the corresponding result of Barbaro-Ceppitelli in [3].

\section{References}

[1] C. Barbaro and R. Ceppitelli, 'Minimax inequalities in Riesz spaces', Atti Sem. Mat. Fis. Univ. Modena 35 (1987), 63-69.

[2] C. Bardaro and R. Ceppitelli, 'Some further generalizations of Knaster-KuratowskiMazurkiewicz theorem and minimax inequalities', J. Math. Anal. Appl. 132 (1988), 484490.

[3] C. Barbaro and R. Ceppitelli, 'Fixed point theorem and vector-valued minimax theorems', J. Math. Anal. Appl. 146 (1990), 363-373.

[4] H. Ben-El-Mechaiekh, P. Deguire and A. Granas, 'Une alternative non lineaire en analyse convexe et applications', C. R. Acad. Sci. Paris Ser. I Math. 295 (1982), 257-259.

[5] H. Ben-El-Mechaiekh, P. Deguire and A. Granas, 'Points fixes et coincidences pour les fonctions multivocues II (Applications de type $\varphi$ et $\varphi^{*}$ ) ', C. R. Acad. Sci. Paris Ser. I Math. 295 (1982), 391-384.

[6] F. E. Browder, 'The fixed point theory of multi-valued mappings in topological vector spaces', Math. Ann. 177 (1968), 283-301.

[7] X. P. Ding, W. K. Kim and K. K. Tan, 'A new minimax inequality on $H$-spaces with applications', Bull. Austrail. Math. Soc. 41 (1990), 457-473.

[8] X. P. Ding, W. K. Kim and K. K. Tan, 'Applications of a minimax inequality on $H$ spaces', Bull. Austral. Math. Soc. 41 (1990), 475-485. 
[9] X. P. Ding and K. K. Tan, 'Matching theorems, fixed point theorems and minimax inequalities without convexity', J. Austral. Math. Soc. (Series A) 49 (1990), 111-128.

[10] X. P. Ding and K. K. Tan, 'Generalizations of $K K M$ theorem and applications to best approximations and fixed point theorems', submitted.

[11] K. Fan, 'Sur un theoreme minimax', C. R. Acad. Sci. Paris Groupe 1, 250 (1964), 39253928.

[12] K. Fan, 'Fixed-point and related thoerems for non-compact convex sets', Game theory and related topics edited by $\mathrm{O}$. Moeschlin and D. Pallaschke, pp. 151-156 (NorthHolland Amsterdam, 1979).

[13] K. Fan, 'Some properties of convex sets related to fixed poin theorems', Math. Ann. 226 (1984), 519-537.

[14] C. A. Ha, 'A non-compact minimax theorem', Pacific J. Math. 97 (1981), 115-117.

[15] C. Horvath, 'Some results on multivalued mappings and inequalities without convexity', Nonlinear and convex analysis edited by B. L. Lin and S. Simons, pp. 96-106 (Marcel Dekker, 1987).

[16] H. M. Ko and K. K. Tan, 'A coincidence theorem with applications to minimiax inequalities and fixed point theorems', Tamkang J. Math. 17 (1986), 37-45.

[17] M. Lassonde, 'One the use of $K K M$ multifunctions in fixed point theory and related topics', J. Math. Anal. Appl. 97 (1983), 151-201.

[18] F. C. Liu, 'A note on the von Neumann-Sion minimax principle', Bull. Inst. Math. Acad. Sinica, 6 (1978), 517-524.

[19] S. Park, 'Generalizations of Ky Fan's matching theorems and their application', J. Math. Anal. Appl. 141 (1989), 164-176.

[20] S. Simons, Two function minimax theorems and variational inequalities for functions on compact and noncompact sets, with some comments on fixed point theorems, (Proc. Sympos. Pure Math. 45(2) (1986), 377-392, Amer. Math. Soc., Providence, R.I.).

[21] M. Sion, 'On nonlinear variational inequalities', Pacific J. Math. 8 (1958), 171-176.

[22] M. H. Shih and K. K. Tan, 'Non-compact sets with convex sections II', J. Math. Anal. Appl. 120 (1986), 264-270.

[23] W. Takahashi, Fixed point, minimax, and Hahn-Banach theorems, (Proc. Sympos. Pure Math. 45(2) (1986), 419-427, Amer. Math. Soc., Providence, R.I.).

Sichuan Normal University Chengdu, Sichuan People's Republic of China 